\title{
Finite-size and surface effects in maghemite nanoparticles: Monte Carlo simulations
}

\author{
Òscar Iglesias and Amílcar Labarta \\ Departament de Física Fonamental, Universitat de Barcelona, Diagonal 647, 08028 Barcelona, Spain
}

(Received 20 December 2000; published 18 April 2001)

\begin{abstract}
Finite-size and surface effects in fine particle systems are investigated by Monte Carlo simulation of a model of a $\gamma-\mathrm{Fe}_{2} \mathrm{O}_{3}$ (maghemite) single particle. Periodic boundary conditions for a large enough system have been used to simulate the bulk properties and the results compared with those for a spherical shaped particle with free boundaries to evidence the role played by the surface on the anomalous magnetic properties displayed by these systems at low temperatures. Several outcomes of the model are in qualitative agreement with the experimental findings. A reduction of the magnetic ordering temperature, spontaneous magnetization, and coercive field is observed as the particle size is decreased. Moreover, the hysteresis loops become elongated with high values of the differential susceptibility, resembling those from frustrated or disordered systems. These facts are a consequence of the formation of a surface layer with higher degree of magnetic disorder than the core, which, for small sizes, dominates the magnetization processes of the particle. However, in contradiction with the assumptions of some authors, our model does not predict the freezing of the surface layer into a spin-glass-like state. The results indicate that magnetic disorder at the surface simply facilitates the thermal demagnetization of the particle at zero field, while the magnetization is increased at moderate fields, since surface disorder diminishes ferrimagnetic correlations within the particle. The change in shape of the hysteresis loops with the particle size demonstrates that the reversal mode is strongly influenced by the reduced atomic coordination and disorder at the surface.
\end{abstract}

DOI: 10.1103/PhysRevB.63.184416

PACS number(s): 75.40.Cx, 05.10.Ln, 75.40.Mg, 75.50.Tt

\section{INTRODUCTION}

The magnetic properties of fine particles are strongly influenced by finite-size and surface effects, their relevance increasing as the particle size decreases. Finite-size effects are due to the nanometric size of the particles, while surface effects are related to the symmetry breaking of the crystal structure at the boundary of each particle. These effects are manifested in nanometric particles through a wide variety of anomalous magnetic properties with respect to those of bulk materials. The magnetic characterization of these systems has put forward the controversial issue of distinguishing between the contributions coming from finite-size and surface effects to their peculiar magnetic properties. For instance, alternately explanations to the reduction of the saturation magnetization $M_{s}$ - a common experimental observation in fine particle systems - has been given in the past. Early models postulated the existence of a so-called dead magnetic layer induced by the demagnetization of the surface spins, which causes a reduction in $M_{s}$ because of its paramagnetic response. In more recent works devoted to the study of different ferrimagnetic oxides $-\gamma-\mathrm{Fe}_{2} \mathrm{O}_{3}, \mathrm{NiFe}_{2} \mathrm{O}_{4}, \mathrm{CoFe}_{2} \mathrm{O}_{4}$, $\mathrm{CuFe}_{2} \mathrm{O}_{4}$, in the form of nanometric particles ${ }^{1-5}$-a random spin canting at the surface, caused by competing antiferromagnetic (AF) interactions, was observed by Mössbauer spectroscopy, ${ }^{2}$ polarized ${ }^{4}$ and inelastic ${ }^{6}$ neutron scattering, and ferromagnetic (FM) resonance. ${ }^{7}$ The origin of this noncollinear arrangement of the spins was discussed by several authors supporting the surface ${ }^{2,8-10}$ or the finite-size explanations, ${ }^{11-15}$ but up to the moment no clear conclusions have been established.

All these ferrimagnetic fine particles share a singular phenomenology at low temperatures. Among the static properties, experiments have shown that the hysteresis loops dis- play high closure fields and do not saturate $e^{14,16,17}$ even at fields of the order of $50 \mathrm{~T}$, which indicates that the anisotropy fields cannot be the only responsible mechanism for the magnetization reversal. Low magnetization as compared to bulk, shifted loops after field cooling and irreversibilities between the field cooling and zero field cooling processes even at high fields are also observed. ${ }^{5,16,17}$ Moreover, the existence of aging phenomena ${ }^{18,19}$ in the time dependence of the magnetization, indicates that there must be some kind of freezing leading to a complex hierarchy of energy levels. Whether these phenomena can be ascribed to intrinsic properties of the particle itself (spin-glass state of the surface which creates an exchange field on the core of the particle ${ }^{14,17}$ ), or they are due to a collective behavior induced by interparticle interactions, ${ }^{20-22}$ has been the object of controversy. ${ }^{23}$

Up to the moment there has been no model giving a clearcut explanation of all the above-mentioned phenomenology, but some works addressing part of the issues have been published in recent years. The first atomic-scale model of the magnetic behavior of individual ferrimagnetic nanoparticles is due to Kodama and Berkowitz. ${ }^{24}$ The authors presented results of calculations of a micromagnetic model of maghemite particles which were based on an energy minimization procedure instead of the Monte Carlo (MC) method. They used Heisenberg spins with enhanced anisotropy at the surface with respect to the core and included vacancies and broken bonds at the surface, arguing that these are indeed necessary to obtain hysteresis loops with enhanced coercivity and high-field irreversibility. Later, Kachkachi et al. ${ }^{25-27}$ performed MC simulations of a maghemite particle described by a Heisenberg model, including exchange and dipolar interactions, using surface exchange and anisotropy constants different to those of the bulk. Their study was mainly focused on the thermal variation of the surface (for 
them consisting of a shell of constant thickness) and core magnetization, concluding that surface anisotropy is responsible for the nonsaturation of the magnetization at low temperatures. No attention was paid, however, to the magnetic properties under a magnetic field.

Other computer simulations studying finite-size and surface effects on ferromagnetic and antiferromagnetic cubic lattices have also been published. Trohidou et al. ${ }^{28,29}$ performed $\mathrm{MC}$ simulations of AF small spherical clusters. By using an Ising model on a cubic lattice, ${ }^{28}$ they computed the thermal and magnetic field dependencies of the magnetization and structure factor, concluding that the particle behaved as a hollow magnetic shell. By means of a Heisenberg model $^{29}$ with enhanced surface anisotropy, they studied the influence of different kinds of surface anisotropy on the magnetization reversal mechanisms and on the temperature dependence of the switching field. Dimitrov and Wysin ${ }^{30,31}$ studied the hysteresis phenomena of very small spherical and cubic FM fcc clusters of Heisenberg spins by solving the Landau-Lifshitz equations. They observed an increase of the coercivity with decreasing cluster size and steps in the loops due to the reversal of surface spins at different fields. However they did not considered the finite temperature effects.

In order to contribute to elucidate the above mentioned experimental controversies and to further develop the previously published numerical simulations, we present the results of a MC simulation of a single spherical particle which aim at clarifying what is the specific role of the finite size and surface on the magnetic properties of the particle, disregarding the interparticle interactions effects. In particular, we will study the magnetic properties under a magnetic field and at finite temperature, thus extending other simulation works. In choosing the model, we have tried to capture the main features of real particles with the minimum ingredients allowing to interpret the results without any other blurring effects.

The rest of the article is organized as follows. In Sec. II we present the model of a maghemite particle upon which the MC simulations are based. In Sec. III, the study of the basic equilibrium magnitudes-energy, specific heat, and magnetization-in absence of magnetic field is presented, comparing results for different particle sizes with those for periodic boundaries. Section IV is devoted to the study of magnetization processes under the presence of a magnetic field. The thermal dependence of hysteresis loops and coercive field are computed, and a detailed analysis of these quantities in terms of the surface and core contributions is performed. The effects of the introduction of different kinds of disorder on the magnetic properties are presented in Sec. $\mathrm{V}$, where we study both the deviation from ideal stoichiometry by random removal of magnetic ions on the whole particle, as well as the introduction of vacancies only at the surface of the particle. In Sec. VI, we end up with a discussion of the obtained results and a presentation of the conclusions.

\section{MODEL}

$\gamma-\mathrm{Fe}_{2} \mathrm{O}_{3}$ maghemite, is one of the most commonly studied nanoparticle compounds ${ }^{24}$ presenting the above-

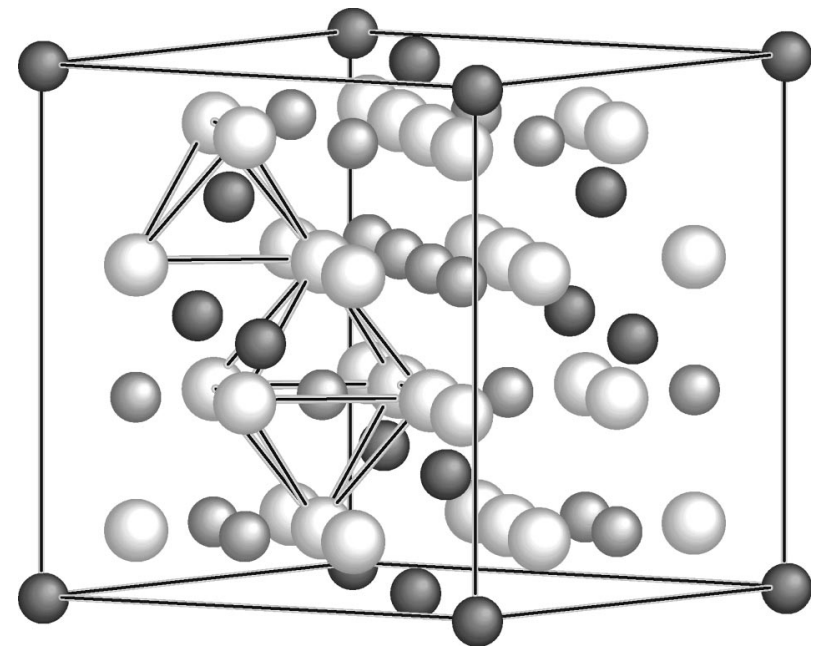

FIG. 1. Unit cell of maghemite. The magnetic $\mathrm{Fe}^{3+}$ ions occupying the two sublattices, in different coordination with the $\mathrm{O}^{2-}$ ions (white color), are colored in black ( $T$ sublattice, tetrahedric coordination) and in grey ( $O$ sublattice, octahedric coordination).

mentioned phenomenology. Maghemite is a ferrimagnetic spinel in which the magnetic $\mathrm{Fe}^{3+}$ ions with spin $S=5 / 2$ are disposed in two sublattices with different coordination with the $\mathrm{O}^{2-}$ ions. Each unit cell (see Fig. 1) has 8 tetrahedric $(T), 16$ octahedric $(O)$ sites, and one sixth of the $O$ sites has randomly distributed vacancies to achieve neutrality charge. The $T$ sublattice has larger coordination than $O$, thus, while the spins in the $T$ sublattice have $N_{T T}=4$ nearest neighbors in $T$ and $N_{T O}=12$ in $O$, the spins in the $O$ sublattice have $N_{O O}=6$ nearest neighbors in $O$ and $N_{T O}=6$ in $T$. In our model, the $\mathrm{Fe}^{3+}$ magnetic ions are represented by Ising spins $S_{i}^{\alpha}$ distributed in two sublattices $\alpha=T, O$ of linear size $N$ unit cells, thus the total number of spin sites is $\left(24 N^{3}\right)$. The choice of Ising spins allows us to reproduce a case with strong uniaxial anisotropy, while keeping computational efforts within reasonable limits. In spite of the fact that this choice could be a crude approximation for moderate anisotropy, which is not the case of maghemite, this will not affect the conclusions of our study, since our main goal is to clarify the effect of the intrinsic magnetic frustration of the maghemite lattice at the surface of the particle. With this aim, we have taken into account the real lattice structure and interactions in the most realistic way. In particular, the possible existence of a spin-glass state at the surface of the particle should be better checked with Ising spins than with a model with continuous spins, since in the former frustration effects are enhanced. ${ }^{32}$ Moreover, the Heisenberg version of the particle without disorder does not show irreversibility in the hysteresis loops, whereas the Ising version does, ${ }^{24,33}$ being easier to observe independently the effects of disorder and finite size in the last case.

The spins interact via antiferromagnetic (AF) exchange interactions with the nearest neighbors on both sublattices and with an external magnetic field $H$, the corresponding Hamiltonian of the model being

$$
\mathcal{H} / k_{B}=-\sum_{\alpha, \beta=T, O} \sum_{i=1}^{N_{\alpha}} \sum_{n=1}^{N_{\alpha \beta}} J_{\alpha \beta} S_{i}^{\alpha} S_{i+n}^{\beta}-h \sum_{\alpha=T, O} \sum_{i=1}^{N_{\alpha}} S_{i}^{\alpha},
$$




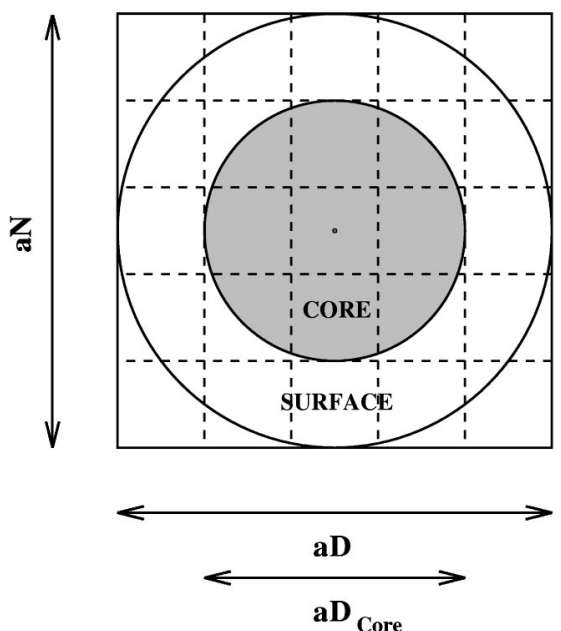

FIG. 2. Schematic drawing of the spherical particles simulated in this study, showing the basic geometric parameters. The unit cells are indicated by the dashed grid, being the cell parameter $a$, and $N$ the number of unit cells along each axis.

where we have defined the field in temperature units as

$$
h=\frac{\mu H}{k_{B}},
$$

$S$ and $\mu$ being the spin value and magnetic moment of the $\mathrm{Fe}^{3+}$ ion, respectively. Hereafter, $S_{i}= \pm 1$ and the maghemite values of the nearest neighbor exchange constants will be considered: ${ }^{24,25} \quad J_{T T}=-21 \mathrm{~K}, \quad J_{O O}=$ $-8.6 \mathrm{~K}, J_{T O}=-28.1 \mathrm{~K}$. Since the intersublattice interactions are stronger than those inside each sublattice, at low temperatures, there must be bulk ferrimagnetic order with spins in each sublattice ferromagnetically aligned and antiparallel intrasublattice alignment.

To simulate the bulk behavior, we have used periodic boundary (PB) conditions for a system of large enough size as to minimize finite-size effects (for systems of linear size $N>8$ these effects are already negligible). When studying finite-size effects, we have considered a spherically shaped particle with $D$ unit cells in diameter and free boundaries (FBs). In the latter case, two different regions are distinguished in the particle: the surface formed by the outermost unit cells and an internal core of diameter $D_{\text {core }}$ unit cells (see Fig. 2). The quantities measured after each MC step are the energy, specific heat, susceptibility and different magnetizations: sublattice magnetizations $\left(M_{O}, M_{T}\right)$, surface and core magnetization $\left(M_{\text {surf }}, M_{\text {core }}\right)$, and total magnetization $\left(M_{\text {total }}\right)$. Each of them have been normalized to the respective number of spins so that they can range from 1 to -1 . In particular, $M_{\text {total }}$ is 1 for ferromagnetic order, 0 for a disordered system and $1 / 3$ for ferrimagnetic order of the $O$ and $T$ sublattices.

The size of the studied particles ranges from $D=3$ to 10 corresponding to real particle diameters from 25 to $83 \AA$ (see Table I). In this table, we have also included the number of surface and core spins $N_{\text {surf }}, N_{\text {core }}$, together with the normalized magnetization values of a ferrimagnetic configuration $M_{\text {unc }}$. Note that due to the finite size of the particles, the
TABLE I. Characteristic parameters of some of the spherical particles simulated: particle diameter $D$ in units of the lattice constant $a$, diameter of the corresponding real $D_{\text {real }}$, number of total spins $N_{\text {total }}$, number of spins at the surface and in the core $N_{\text {surf }}, N_{\text {core }}$, and magnetization of the noncompensated spins $M_{\text {unc }}$ $=\left(N_{O}-N_{T}\right) / N_{\text {total }}$. The data are for particles with no vacancies in the $O$ sublattice.

\begin{tabular}{lcccccc}
\hline \hline$D$ & $D_{\text {real }}(\AA)$ & $N_{\text {surf }}$ & $N_{\text {core }}$ & $M_{\text {unc }}^{\text {surf }}$ & $M_{\text {unc }}^{\text {core }}$ & $M_{\text {unc }}$ \\
\hline 3 & 25 & $330(95 \%)$ & 17 & 0.285 & 0.412 & 0.291 \\
4 & 33 & $724(87 \%)$ & 111 & 0.337 & 0.369 & 0.341 \\
5 & 41 & $1246(78 \%)$ & 347 & 0.355 & 0.291 & 0.341 \\
6 & 50 & $1860(69 \%)$ & 841 & 0.350 & 0.332 & 0.344 \\
8 & 66 & $3748(58 \%)$ & 2731 & 0.345 & 0.330 & 0.338 \\
10 & 83 & $6485(48 \%)$ & 12617 & 0.329 & 0.337 & 0.333 \\
\hline \hline
\end{tabular}

ratio of $T$ and $O$ spins produces $M_{\text {unc }}$ values that, in general, do not coincide exactly with the $1 / 3$ value for perfect ferrimagnetic order in an infinite lattice. In order to make the measured magnetizations for different diameters comparable, we have normalized them to the corresponding $M_{\text {unc }}$ values given in Table I.

\section{EQUILIBRIUM PROPERTIES}

\section{A. Energy and specific heat}

We start by studying the effect of free boundary conditions and finite-size effects on the equilibrium properties in zero magnetic field. The simulations have been performed using the standard Metropolis algorithm. Starting from a high enough temperature $(T=200 \mathrm{~K})$ and an initially disordered state with spins randomly oriented, the system was cooled down at a constant temperature step $\delta T=-2 \mathrm{~K}$ and, after discarding the first $1000 \mathrm{MC}$ steps in order to allow the system to thermalize, the thermal averages of the thermodynamic quantities were computed at each temperature during a number of MC steps ranging from 10000 to 50000 depending on the system size. The starting configuration at each new temperature was the one obtained at the end of the averaging process at the previous temperature. Systems with periodic and free boundary conditions with spherical shape have been considered with sizes ranging from 3 to 14 .

In Fig. 3, we compare the thermal dependence of the energy for spherical particles of different diameters $D$ with the corresponding results for a system of size $N=14$ and $\mathrm{PB}$ (lowermost curve, left triangles). A second order transition from paramagnetic to ferrimagnetic order signaled by a sharp peak at $T_{c}(D)$ in the specific heat (see the inset in Fig. 3) is clearly observed. Finite size effects on both the energy and the specific heat are very important even for $D$ 's as large as 14 in the FB case, while for PB conditions they are negligible already for $N=8$. The energy difference between the disordered and ferrimagnetic phases as well as the critical temperature $T_{c}(D)$ increases as $D$ is increased. This last quantity is strongly size dependent and approaches the infinite size limit $\left(T_{c}(\infty)=126 \pm 1 \mathrm{~K}\right.$ as evaluated for the $N$ $=14$ system with PB conditions) as $D$ increases (see Fig. 4, 


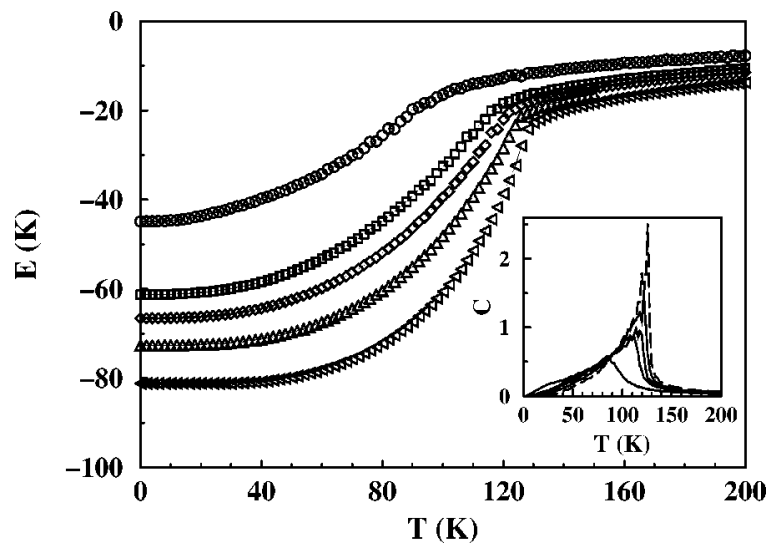

FIG. 3. Thermal dependence of the energy for different diameters $D=3,6,8,14$ (from the uppermost curve) and periodic boundary conditions $N=14$ (lowermost curve). Inset: Thermal dependence of the specific heat for the same cases (the periodic boundary case is drawn with a dashed line).

in which the variation of the peak in the specific heat with $1 / D$ has been plotted). $T_{c}(D)$ can be fitted to the scaling law

$$
\frac{T_{c}(\infty)-T_{c}(D)}{T_{c}(\infty)}=\left(\frac{D}{D_{0}}\right)^{-1 / \nu}
$$

as predicted by finite-size scaling theory ${ }^{34,35}$ with $D_{0}=1.86$ \pm 0.03 a microscopic length scale (in this case, it is roughly twice the cell parameter), and a critical exponent $\nu=0.49$ \pm 0.03 , which seems to indicate a mean field behavior. ${ }^{36}$ This result can be ascribed to the high coordination of the $O$ and $T$ sublattices. The fitted curve is drawn in Fig. 4 where deviations from scaling are appreciable for the smallest diameters for which corrections to the finite-size scaling of Eq. (3) may be important. ${ }^{34}$

\section{B. Magnetization}

To study the effects of a free surface and of finite size on the magnetization of the particles, we compare in Fig. 5 the results for four particle diameters ( $D=3,4,6,8$, open circles)

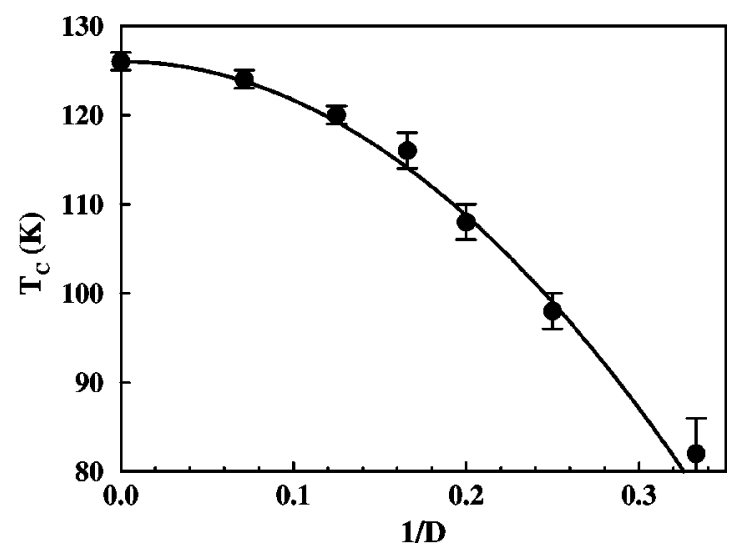

FIG. 4. Particle size dependence of the transition temperature $T_{c}$ from paramagnetic to ferrimagnetic phases for spherical particles with FB. The displayed values have been obtained from the maximum in the specific heat. The continuous line is a fit to Eq. (3). with that corresponding to a $N=14$ system with PB (representing the behavior of the bulk). In this figure, we have distinguished the surface (dashed lines) and core (dot-dashed lines) contributions to the total magnetization (symbols). The results have been recorded during the same cooling procedure used to obtain the energy. The main feature observed is the reduction of the total magnetization $M_{\text {total }}$ with respect to the PB case (continuous line) due to the lower coordination of the spins at the surface, which hinders ferrimagnetic order at finite temperatures. Figure 5 clearly shows the roles played by the surface and the core in establishing the magnetic order. On one hand, independently of the particle size, the core (dot-dashed lines) tends to a perfect ferrimagnetic order at low $T$ (marked by $M=1 / 3$ ), progressively departing from the bulk behavior as $T$ approaches $T_{c}$, this finite-size effect being more important as the particle size decreases. However, the surface magnetization does not attain perfect ferrimagnetic order at $T=0$ even for $D=8$ due to the reduced coordination of the spins. For this reason, a rapid thermal demagnetization is observed which significantly departs $M_{\text {surf }}$ from the bulk behavior.

It is worthwhile to note that for all the diameters studied there is a temperature range in which this demagnetization process is linear, this range being wider as the particle size decreases. In this linear regime, the particle demagnetization becomes dominated by the surface effects, being the core and surface behaviors strongly correlated. Linear demagnetization is indicative of the effective 3D-2D dimensional reduction of the surface shell and has previously been observed in thin film systems ${ }^{37,38}$ and in simulations of rough FM surfaces ${ }^{39} M_{\text {total }}$ is always strongly dominated by the surface contribution, progressively tending to the bulk behavior as the particle size is increased.

In Fig. 6 we show the size dependence of the $M_{\text {total }}$ at different temperatures. All the curves follow a quasilinear behavior with $1 / D$ except for very small particle sizes $(D$ $=3$ ). This is consistent with the existence of a surface layer of constant thickness $\Delta r$ independently of $D$ and with reduced magnetization with respect to the core. With these assumptions, the size dependence of $M$ can be expressed as

$$
M(D)=M_{\text {core }}-\Delta M \frac{\Delta r S}{V}=M_{\text {core }}-\Delta M \frac{6 \Delta r}{D},
$$

where $S$ and $V$ are the surface and volume of the particle, and $\Delta M=M_{\text {core }}-M_{\text {surface }}$.

\section{HYSTERESIS LOOPS}

In Fig. 7, we show the hysteresis loops of particles with diameters $D=3,6$ for different temperatures. The loops have been computed by starting from a demagnetized state at $h$ $=0$ and increasing the magnetic field in constant steps, $\delta h$ $=1 \mathrm{~K}$, during which the magnetization was averaged over $\approx 3000$ MC steps after thermalization. The results shown have been averaged for several independent runs starting with different random seeds.

First of all, let us note that the saturation field and the high field susceptibility increase as the particle size is re- 

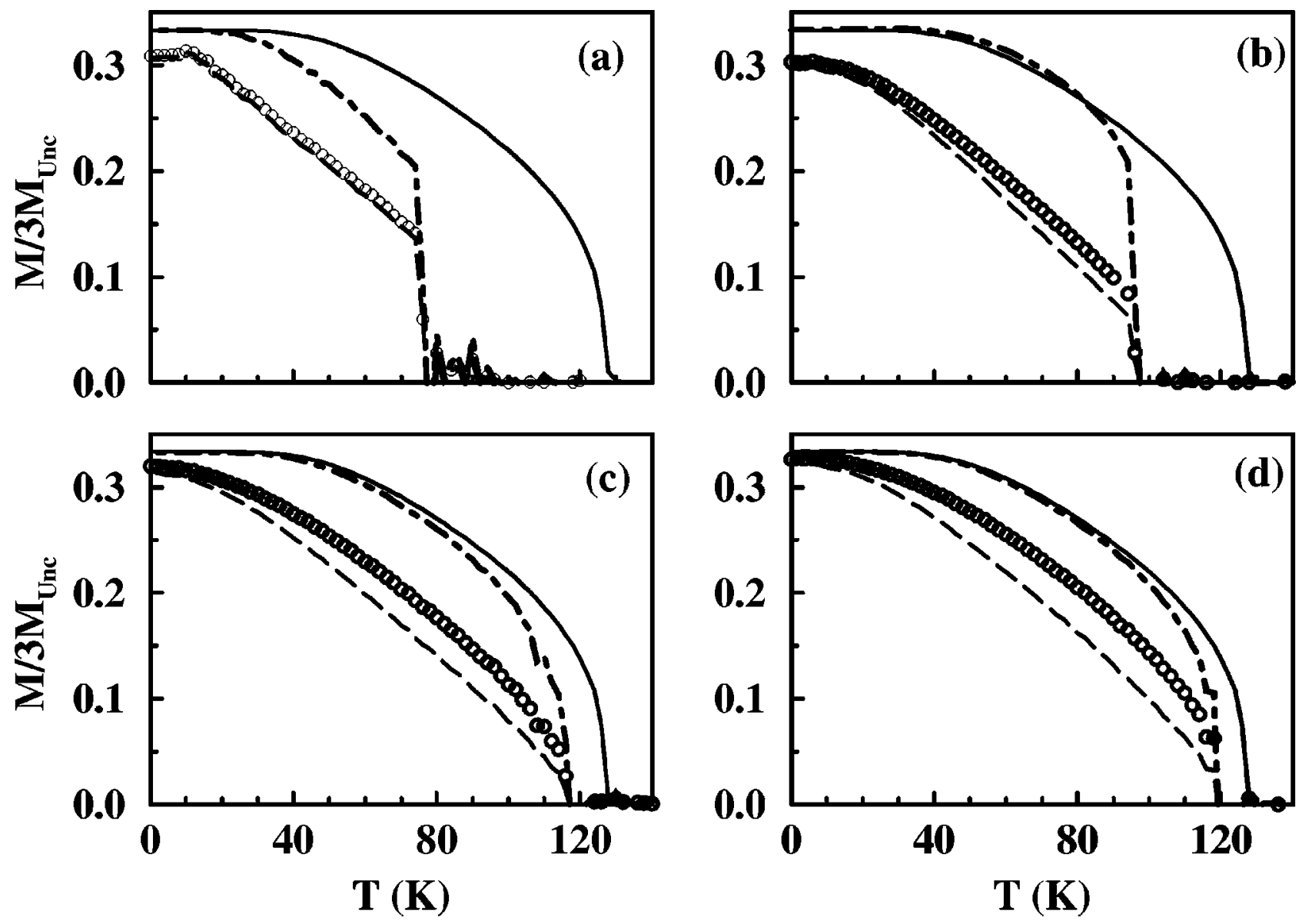

FIG. 5. Thermal dependence of the magnetization $M$ obtained by progressive cooling from high $T$ at a constant rate, $\delta T=-2 \mathrm{~K}$, and starting from a random configuration of spins. The results for four particle diameters are shown: $D=3$ (a), $D=4$ (b), $D=6$ (c), and $D$ $=8$ (d). The contributions of the surface (dashed line) and core spins (dot-dashed line) have been distinguished from the total magnetization (circles). The results for PB conditions, in a system of linear size $N=14$, have also been included for comparison (continuous line).

duced, since this quantities are mainly associated to the progressive alignment of the surface spins towards the field direction. Thus, the loops of the smallest particles resemble those found in ferrimagnetic nanoparticles ${ }^{3,14,24}$ and other bulk systems with disorder, ${ }^{32,40}$ increasing their squaredness (associated to the reversal of $M$ as a whole) with the size. In fact, by plotting separately the contributions of the core and the surface to the total magnetization (see Fig. 8, dashed lines), we see that the loop of the core is almost perfectly squared independently of temperature and particle size, indicating a reversal of its magnetization with a well-defined ferrimagnetic moment. Instead, the loop of the surface reveals a progressive reversal of $M$, which is a typical feature associated to disordered or frustrated systems. ${ }^{32,40}$ Nonetheless, for a wide range of temperatures and particle sizes, it is the reversal of the surface spins which triggers the reversal of the core. This is indicated by the fact that the coercive field of the core is slightly higher but very similar to the one of the surface.

Since for all the studied particle sizes the $h_{c}(T)$ curves show a complex behavior mainly related to the frustration of the antiferromagnetic intra and intersublattice exchange interactions, we start by studying the case of a ferromagnet with no frustration. In Fig. 9(a), the $h_{c}(T)$ dependence for a system with $N=8$ and with the same lattice structure as maghemite but equal FM interactions $J_{\alpha \beta}=J$ and PB conditions is shown. The $h_{c}(T)$ dependence is now a monotonously decreasing curve with no inflection point, which at high enough temperatures $(T / J \gtrsim 1)$ can be fitted to a power law of the kind

$$
h_{c}(T)=h_{c}(0)\left[1-\left(T / T_{c}\right)^{1 / \alpha}\right] \text {, }
$$

with $\alpha=2.26 \pm 0.03$; close but different to what would be obtained by a model of uniform reversal such as Stoner-Wohlfarth $^{41}(\alpha=2)$. Even in this simple case, for which $M$ reverses as a whole, the thermal variation of $h_{c}(T)$ cannot be only ascribed to the thermal activation of a constant magnetization vector over an energy barrier landscape, since actually $M$ is of course temperature dependent. Therefore, the reversal mechanism cannot be inferred from the $\alpha$ value obtained from a fit to Eq. (5) in any range of temperatures for which $M$ significantly varies with $T$.

The thermal dependence of $h_{c}$ for the maghemite particles with AF interactions is shown in Fig. 9(b). Both for the PB and spherical cases, the $h_{c}(T)$ curves are qualitatively different from the FM case: they have opposite curvature and two regimes of thermal variation. 


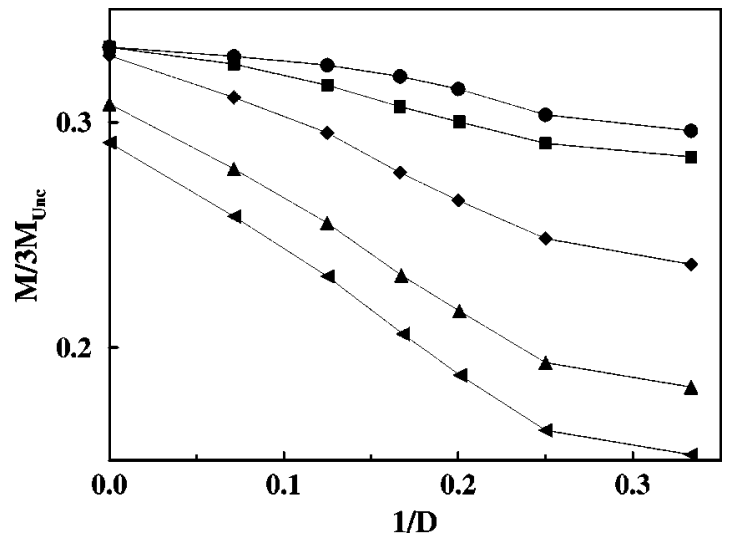

FIG. 6. Size dependence of the magnetization of a spherical particle at different temperatures $T=0,20,40,60,70 \mathrm{~K}$ (from upper to lowermost curves).

Let us start by analyzing the PB case. At high $T$ ( $T$ $\geq 20 \mathrm{~K}), h_{c}(T)$ can be fitted to the power law of Eq. (5) with $\alpha=0.94 \pm 0.02, h_{c}(0)=134 \pm 2 \mathrm{~K}$. Values of $\alpha$ close to 1 have been deduced in the past for some models of domain wall motion. ${ }^{42}$ At low $T$, a different regime is entered but tending to the same $h_{c}(0)=134.2 \mathrm{~K}$. This change in behavior is associated to the wandering of the system through metastable states with $M_{\text {total }} \simeq 0$, which are induced by the frustration among AF interactions. Consequently, when low-
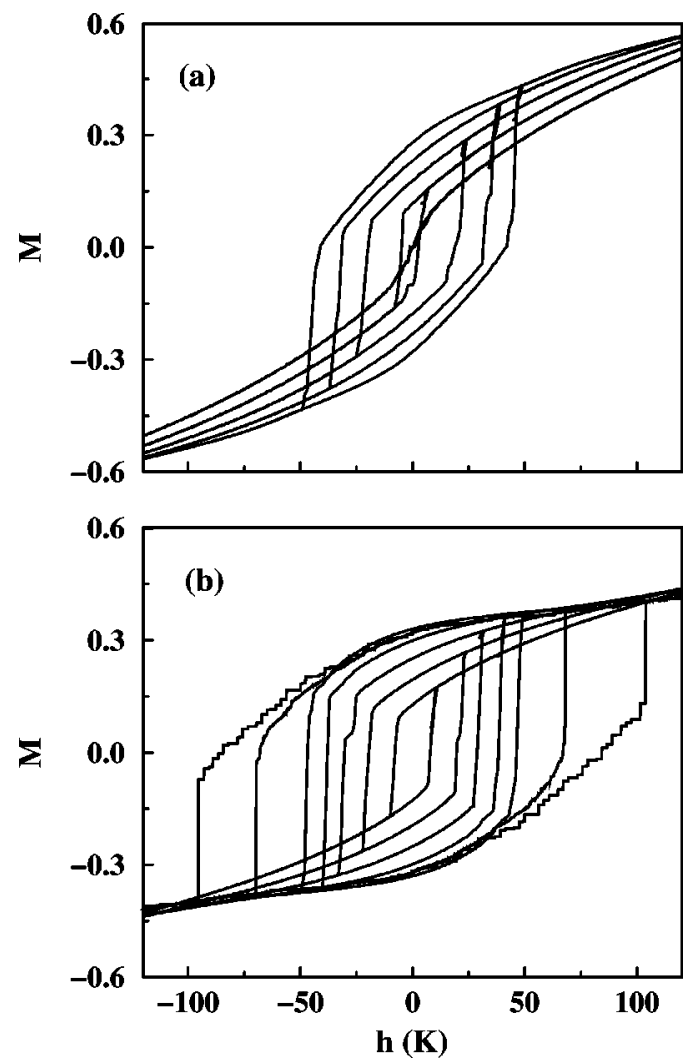

FIG. 7. Temperature dependence of the hysteresis loops for particles of diameter $D=3$ (a), $D=6$ (b). The temperatures starting from the outermost loop are $T=0,5,20,40,60,80,100 \mathrm{~K}$, except for case $D=3$ in which $T=0,5 \mathrm{~K}$ curves are omitted for clarity.
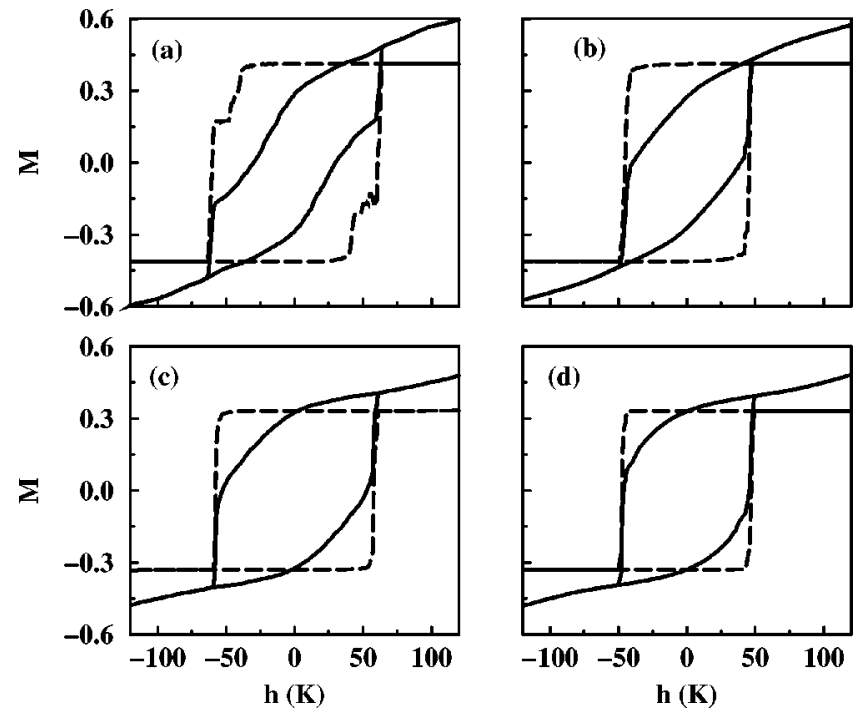

FIG. 8. Surface (continuous line) and core (dashed line) contributions to the hysteresis loops for particles of diameters $D=3, T$ $=10 \mathrm{~K}(\mathrm{a}) ; D=3, T=20 \mathrm{~K}(\mathrm{~b}) ; D=6, T=10 \mathrm{~K}(\mathrm{c}) ; D=6, T$ $=20 \mathrm{~K}(\mathrm{~d})$.
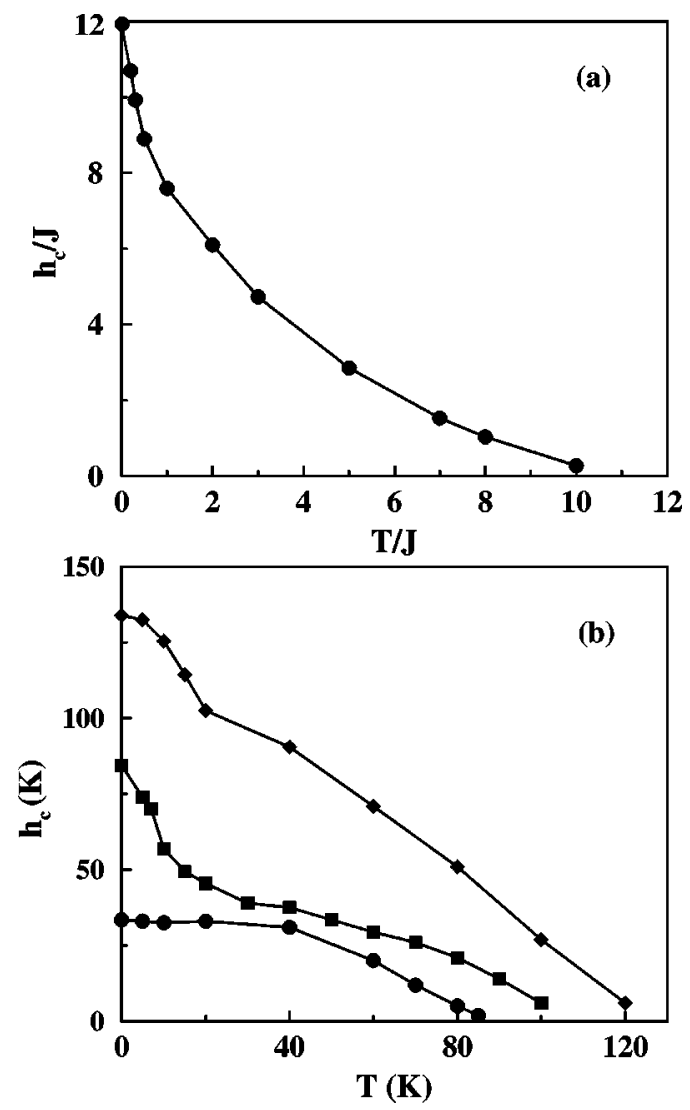

FIG. 9. (a) Temperature dependence of the coercive field $h_{c}$ for a system with the same structure as maghemite but ferromagnetic interactions $\left(J_{\alpha \beta}=J\right)$ and PB conditions and $N=8$; (b) Temperature dependence of the coercive field $h_{c}$ for the real AF values of the exchange constants for maghemite for the case of FB spherical particles of diameters $D=3$ (circles), $D=6$ (squares), and for a system of linear size $N=8$ with $\mathrm{PB}$ conditions (diamonds). 


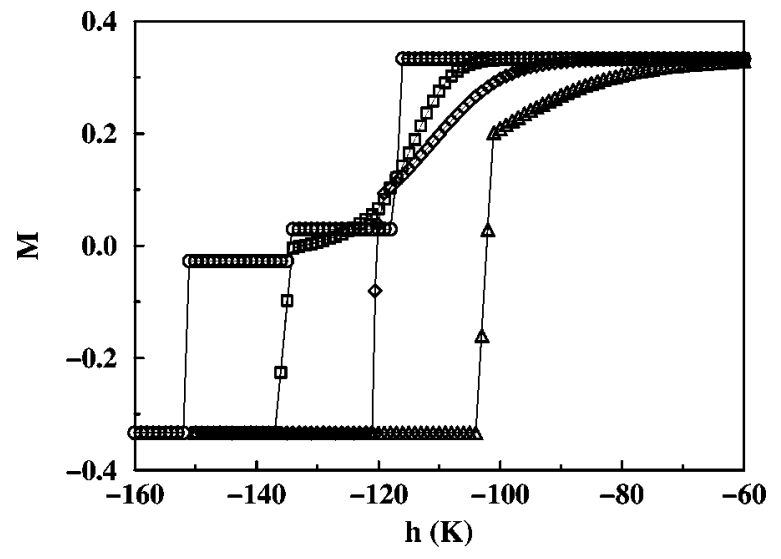

FIG. 10. Detail of low temperature hysteresis loops for PB conditions around the coercive field $h_{c}$. The corresponding temperatures are $T=0$ (circles), $5 \mathrm{~K}$ (squares), $10 \mathrm{~K}$ (diamonds), $20 \mathrm{~K}$ (triangles).

ering $T$, the hysteresis loops become steplike around $h_{c}$ as shown in Fig. 10 (similar features are observed in related studies $\left.{ }^{30,31,43}\right)$. The jumps at $T=0$ are located at $h$ $=117,134.2,151.4 \mathrm{~K}$, the values at which the magnetic field energy is enough to invert one $O$ spin having 0,1,2 $O$ nearest neighbors inverted, respectively. ${ }^{43}$ While the $O$ sublattice reverses progressively, the $T$ sublattice, instead, reverses as a whole after the reversal of $O$, at $h=151.4 \mathrm{~K}$. When $T$ is increased from 0 , the steps are rounded by the progressive population of states with greater degree of configurational disorder and less metastability, finally giving rise to the suppression of the steps for $T$ around $12 \mathrm{~K}$, when $h_{c} \lesssim 117 \mathrm{~K}$ and the high $T$ regime of $h_{c}(T)$ is entered.

The general $h_{c}(T)$ behavior for spherical particles with FB strongly depends on the particle size. For $D=3,6$ and $T \gtrsim 20 \mathrm{~K}$, the $h_{c}$ decay is similar to that for $\mathrm{PB}$, but, at any given $T$, being smaller than for $\mathrm{PB}$ and as the size of the particle is decreased. At these temperatures, $h_{c}(T)$ is dominated by the surface, which nucleates the reversal of the magnetization, as indicated by the proximity between the surface and core $h_{c}$ (see Fig. 8). However, when lowering $T$ below $20 \mathrm{~K}, M_{\text {surf }}$ and $M_{\text {core }}$ tend to be equal, the surface becomes less efficient as nucleation center for spin reversal, and $h_{c}$ becomes dominated by the core $\left(h_{c}^{\text {surf }}<h_{c}^{\text {core }}\right.$ for any particle size, see Fig. 8). This is the cause of the rapid increase of $h_{c}$ towards the PB values for the $D=6$ curve [see Fig. 9(b)]. For $D=3$, instead, $h_{c}$ saturates when lowering $T$ due to the smaller ratio of core to surface spins, which actually hinders the prevalence of the core.

Finally, it is worth noticing that, independently of the size of the particles with $\mathrm{FB}$, the $h_{c}$ values are always smaller than that for $\mathrm{PB}$, since the existence of spins with less coordination at the surface favors the formation of reversed nuclei of spins acting as a seed for the reversal process, which is not the case of $\mathrm{PB}$, where all equivalent spins have the same coordination. Therefore, the $h_{c}$ values for PB are only recovered at low $T$ in the limit of large particle size, at difference with other extensive magnitudes such as the energy or the magnetization, for which we have checked that finitesize scaling is accomplished.
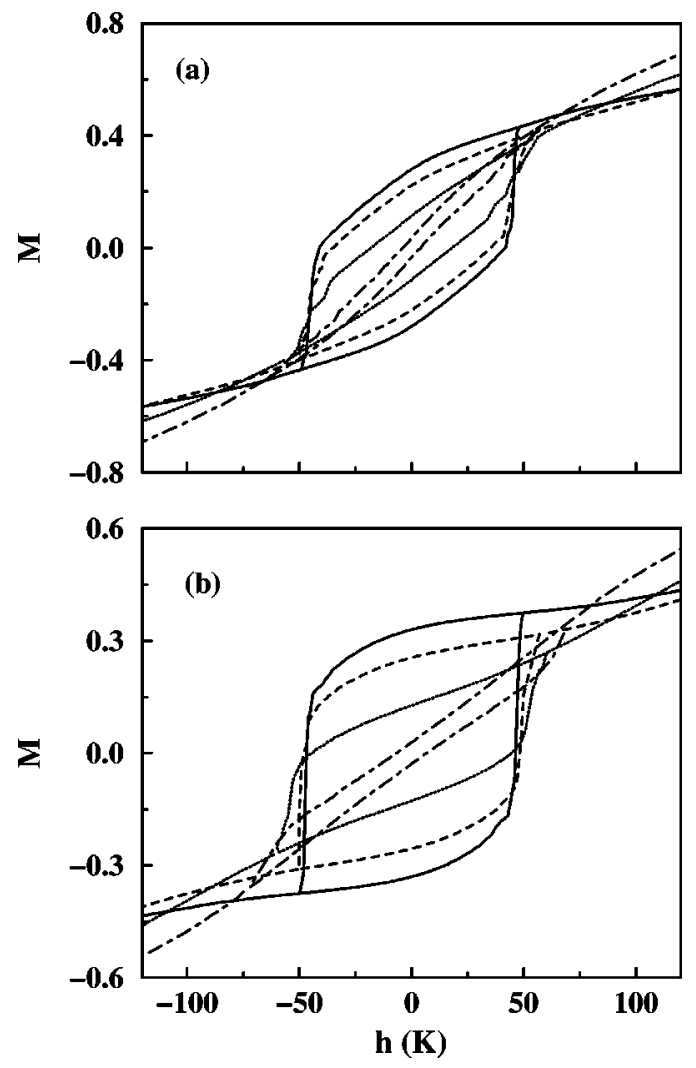

FIG. 11. Hysteresis loops for systems with vacancy concentrations $\rho_{v}=0.0,0.166,0.4,0.6$ (from outer to innermost) on the $O$ sublattice at $T=20 \mathrm{~K}$. Particle diameters $D=3$ (a) and $D=6$ (b). Results have been averaged over 10 disorder realizations.

\section{EFFECTS OF DISORDER}

In real particles, disorder and imperfections are present departing the system from perfect stoichiometry and distort the position of the atoms on the lattice, being their effect more important at the surface. ${ }^{15}$ There are several ways to implement this disorder on the model. The simplest way to simulate the deviation of the $O$ and $T$ sublattice atoms from ideal stoichiometry is by random removal of magnetic ions on the $O / T$ sublattices.

\section{A. Disorder on the lattice}

Up to the moment, the existence of vacancies in the $O$ sublattice in real maghemite structure has not been considered. It is important to note that, in this system, intrasublattice and intersublattice magnetic interactions are antiferromagnetic. Consequently, inclusion of vacancies in one of the sublattices may destabilize the FM parallel alignment of the other one, resulting in a system with a great degree of magnetic disorder. In particular, this effect will be much stronger when vacancies are introduced in the $O$ sublattice, since $N_{T O}$ is greater than $N_{T O}$. To show the effect of these kind of disorder, we have simulated the hysteresis loops for different vacancy concentrations $\rho_{v}$ on the $O$ sublattice at two cooling fields $h_{\mathrm{FC}}=20,100 \mathrm{~K}$. As can be seen in Fig. 11, the introduction of a low concentration of vacancies $\left(\rho_{v}=1 / 6\right.$ as in the real material) results in a reduction of the magnetization 

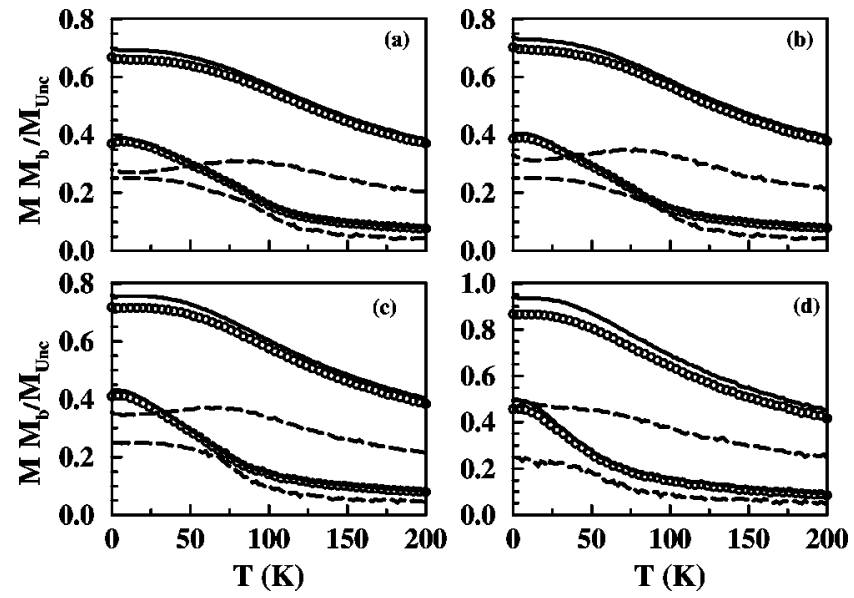

FIG. 12. Thermal dependence of $M$ after cooling under a magnetic field for a spherical particle with $D=3$, with vacancy densities on the surface of the $O$ and $T$ sublattices $\rho_{s v}=0$ (a), 0.1 (b), 0.2 (c), $0.5(\mathrm{~d})$, and $\rho_{v}=0.166$ on the $\mathrm{O}$ sublattice. The results for two cooling fields $h_{\mathrm{FC}}=20,100 \mathrm{~K}$ (lower and upper curves, respectively, in each panel) are shown. The contributions of the surface (thick lines) and the core (dashed lines) to the total magnetization (circles) have been plotted separately. The magnetization has been normalized to $M_{b}$, the magnetization of a perfect ferrimagnetic configuration for a system of infinite size.

and increases the high field susceptibility without any substantial change in the general shape of the loops. However, if $\rho_{v}$ is increased beyond the actual value, the loops progressively closes, loosing squaredness and progressively resembling those for a disordered ${ }^{32,40}$ system, with high values of the high field susceptibilities and much lower coercivity.

\section{B. Surface disorder}

In what follows, we will study the effects of the disorder at the surface of the particle, considering a $\rho_{v}=1 / 6$ vacancy density on the $O$ sublattice. Since the surface of the particles is not an ideal sphere, the outermost unit cells may have an increased number of vacancies on both sublattices with respect to those present in the core. Reduced coordination at the surface may also change the number of links between the surface atoms. We will denote by $\rho_{s v}$ the concentration of surface vacancies in the outermost primitive cells.

\section{Field coolings}

The magnetic ordering of the system can be characterized by studying the behavior of the equilibrium magnetization in a magnetic field. These curves have been obtained by the same cooling procedure used in the magnetization simulations at zero field with $\delta T=-2 \mathrm{~K}$ in presence of different cooling fields $h_{\mathrm{FC}}$. Several such curves are shown in Figs. 12,13 , in which the surface (continuous lines) and the core (dashed lines) contributions to the total magnetization (open symbols) have been distinguished. Let us first analyze the case with no surface disorder $\left(\rho_{s v}=0\right)$. The curves at different cooling fields do not collapse to the perfect ferrimagnetic order value at low $T$ (i.e., $M_{\text {total }}=1 / 4$ for $\rho_{s v}=1 / 6$ ), reaching higher values of the magnetization the higher $h_{\mathrm{FC}}$, being this
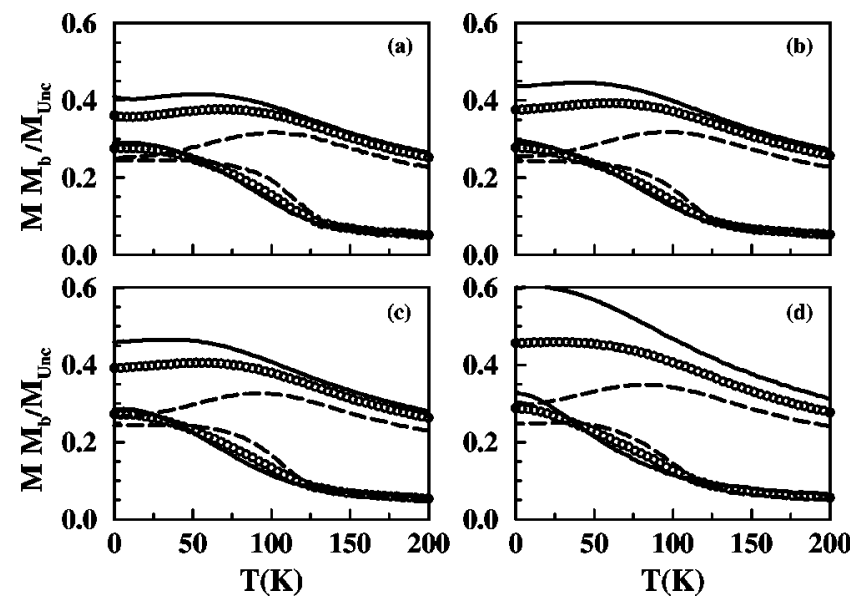

FIG. 13. Same as Fig. 11 but for a spherical particle of diameter $D=6$.

effect greater as the particle size is reduced [compare Figs. 12(a) and 13(a)]. This is in contrast with the results for PB (not shown), for which the system reaches perfect ferrimagnetic order at low $T$, even at fields higher than $100 \mathrm{~K}$, evidencing that the main effect of the surface is the breaking of ferrimagnetic correlations within the particle. As a consequence, at a given temperature, the FM order induced by a magnetic field increases when decreasing $D$.

By separately analyzing in detail the behavior of the surface and core contributions to the total magnetization, deeper understanding of finite-size effects can be gained. As in the case of $h=0$, the total magnetization for small particles is completely dominated by the surface contribution (continuous lines in Figs. 12,13) and this is the reason why the ferrimagnetic order is less perfect at these small sizes and the magnetic field can easily magnetize the system. However, the behavior of the core of the smallest particles is still very similar to that of the case with $\mathrm{PB}$, although its contribution to $M_{\text {total }}$ is very small. At low fields, the surface is always in a more disordered state than the core: its magnetization lies below $M_{\text {total }}$ at temperatures for which the thermal energy dominates the Zeeman energy of the field [see the continuous lines in Figs. 12(a), 13(a)]. In this regime, the total magnetization closely follows that of the surface [see the curves in Figs. 12(a) and 13(a) for $h_{\mathrm{FC}}=20 \mathrm{~K}$ ] for the two simulated sizes. In contrast, a high field is able to magnetize the surface easier than the core due to the fact that the broken links at the surface worsen the ferrimagnetic order, while the core spins align towards the field direction in a more coherent way. Only for the biggest particles the surface contribution departs from the $M_{\text {Total }}$ indicating the increasing contribution of the core (see the curves in Figs. 12a and 13a for $h_{F C}=20 \mathrm{~K}$ ). Note also that, in this high $h_{\mathrm{FC}}$ regime, a maximum appears which is due to the competition between the FM alignment induced by the field and the spontaneous ferrimagnetic order (as the temperature is reduced the strength of the field is not enough as to reverse the spins into the field direction).

The introduction of vacancies does not change the low field behavior of the total magnetization, which is still dominated by the surface both for $D=3,6$, although the smallest particles are easily magnetized by the field. However, at high 

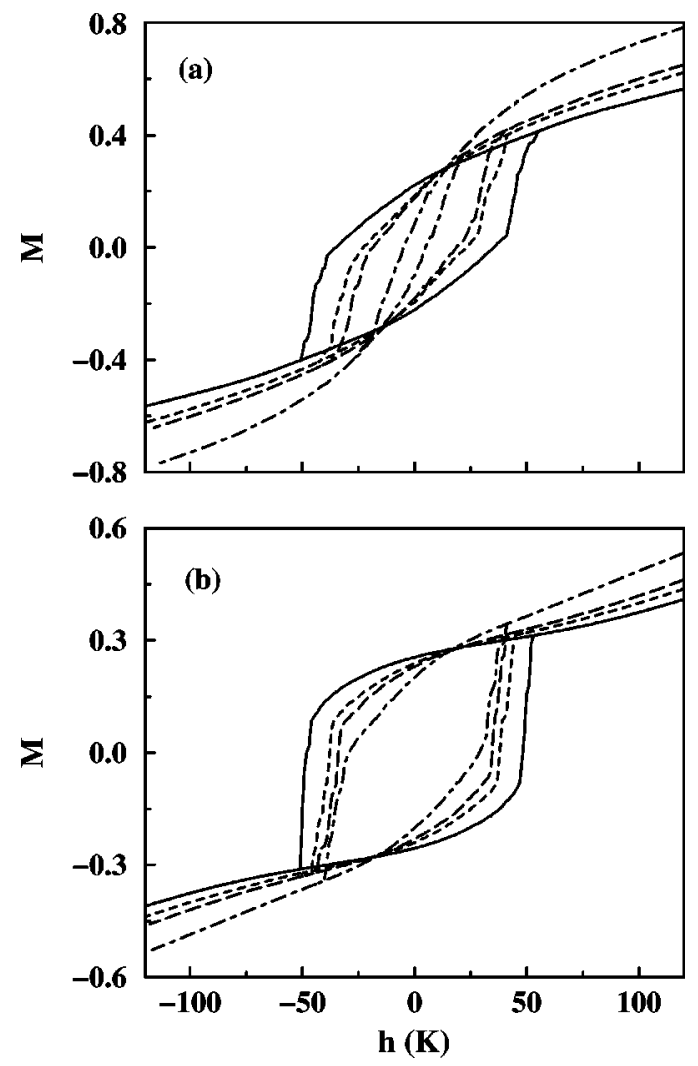

FIG. 14. Hysteresis loops for systems with vacancy densities on the surface of the $O$ and $T$ sublattices $\rho_{s v}=0,0.1,0.2,0.5$, vacancy density $\rho_{s v}=0.1666$ on the $O$ sublattice, and $T=20 \mathrm{~K}$. Particle diameters $D=3$ (a), $D=6$ (b). Results have been averaged over ten disorder realizations.

fields, $M_{\text {total }}$ is lower than $M_{\text {surf }}$, the surface progressively decouples from $M_{\text {total }}$ with the introduction of vacancies in the surface, being this effect more remarkable for the biggest particle. With respect to the core, at difference with the non disordered case $\left(\rho_{v}=\rho_{s v}=0\right)$, the low temperature plateau of $M_{\text {core }}$ tends to a higher value than that for perfect ferrimagnetic order, since the main effect of the disorder is to break ferrimagnetic correlations in the core; increasing the ferromagnetic order induced by the field. This is reflected in a progressive departure of the high and low field $M_{\text {core }}$ curves with increasing disorder [see the dashed lines in the sequence (b)-(d) of Figs. 12, 13]. The maximum appearing at high $h_{\mathrm{FC}}$ is only slightly affected by disorder, shifting to lower temperatures and eventually disappearing for $D=3$ and $\rho_{s v}=0.5$.

\section{Hysteresis loops}

Hysteresis loops with surface disorder are given in Fig. 14 for two particle diameters. The introduction of surface vacancies facilitates the magnetization reversal by progressive rotation, producing a rounding of the hysteresis loops when approaching $h_{c}$, in the same way that occurs when particle size is reduced. The same fact explains the increase of the high field susceptibility, since the vacancies act as nucleation centers of FM domains at the surface, which, from there on,
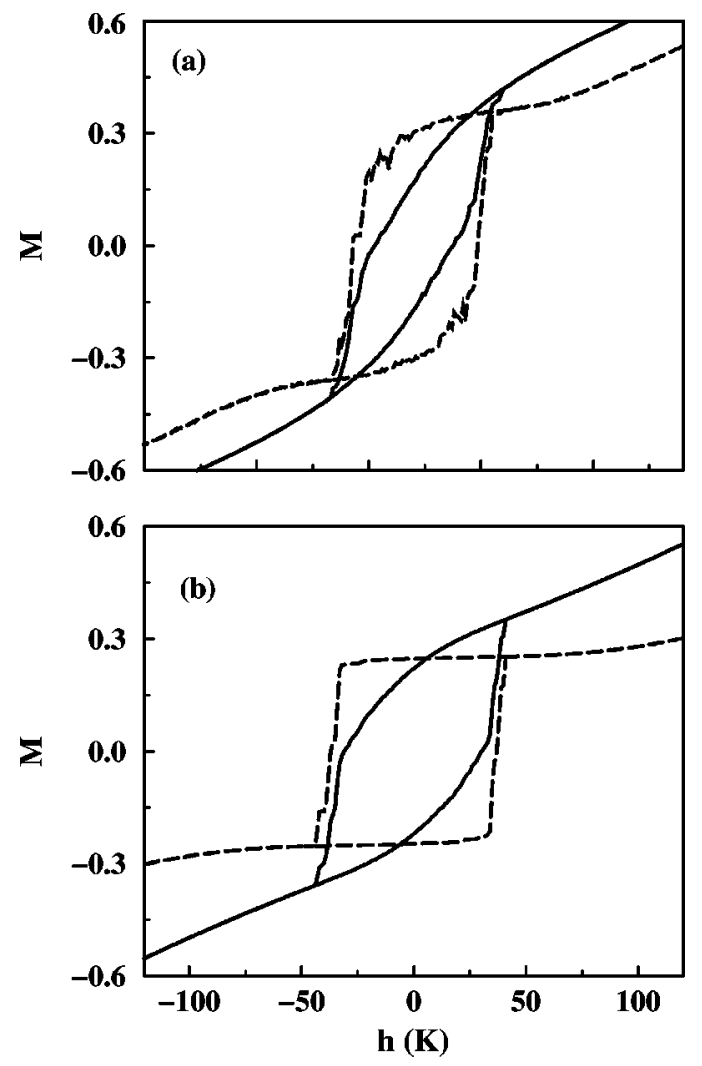

FIG. 15. Core (dashed lines) and surface (solid lines) contributions for the case $\rho_{s v}=0.2$ of Fig. 13 .

extend the FM correlations to the inner shells of spins. Moreover, a considerable decrease of $h_{c}$ is observed. All these facts yield to a progressive elongation of the loops, giving loop shapes resembling those of disordered systems. ${ }^{32,40}$ Figure 15 , where the surface and core contributions are shown separately, clearly evidences that the increase of FM correlations at the surface, facilitated by the vacancies, induce FM order in the core. That is to say, $M_{\text {core }}$ follows the evolution of $M_{\text {surf }}$ at moderate fields above $h_{c}$, in contrast with the case with no surface vacancies (see Fig. 8) where the core keeps the ferrimagnetic order for the same field range.

\section{DISCUSSION AND CONCLUSIONS}

We have presented a simple model of a maghemite nanoparticle with the minimal ingredients necessary to faithfully reproduce the magnetic structure of the real material. The model has proven successful in reproducing several key features present in ferrimagnetic nanoparticle systems: (1) the reduction of $T_{c}$, spontaneous magnetization $M_{\text {total }}$, and coercive field $h_{c}$, for small sizes, as $D$ decreases, (2) the increase, with the reduction of the particle size and with the increase of surface disorder, of the differential susceptibility and the elongation of the hysteresis loops in resemblance with those of frustrated systems, and (3) the existence of a surface layer with higher magnetic disorder than the core. Let us comment on these points in deeper detail.

First of all, we find that $T_{c}(D)$ follows conventional finite-size scaling, discarding any important surface effect on 
this quantity. Similar finite-size effects have been found in fine particles ${ }^{44}$ of $\mathrm{MnFe}_{2} \mathrm{O}_{4}$, but with a surprising increase of $T_{c}(D)$ as $D$ decreases. However, the spontaneous magnetization $M_{\text {total }}$, at any temperature, follows a quasilinear behavior with $1 / D$, see Fig. 6, indicating that the reduction of $M_{\text {Total }}$ is simply proportional to the ratio of surface to core spins, so it is mainly a surface effect. Similar experimental behavior has been found in $\gamma-\mathrm{Fe}_{2} \mathrm{O}_{3}$ (Ref. 45) and the above mentioned system. ${ }^{44}$

The $h_{c}(T)$ thermal decay for the spherical particles is in qualitative agreement with the experimental results for maghemite particles of sizes $9-10 \mathrm{~nm}$ shown in Fig. 4 of Ref. 17, taking into account that in real samples there are additional contributions coming from the blocking process associated to the particle size distribution ( $h_{c}$ drops to zero above the blocking temperature). In both cases, the curvature of the $h_{c}(T)$ curve is similar, suggesting a progressive reversal of the magnetization, a point that is also confirmed by the shape of the hysteresis loops around $h_{c}$. However, our model for spherical particles gives reduced coercivities with respect to the bulk (represented by the PB case). A fact that is in contrast with the enhancement observed experimentally, ${ }^{14,17,20}$ and indicating that finite-size effect cannot cause it. Increased anisotropy at the surface may be the responsible for it. In any case, the model qualitatively reproduces the $h_{c}$ reduction with $D$ for small sizes (see Fig. 1 in Ref. 14), which may be indeed a finite-size effect.

The $M(T)$ and $M(h)$ dependencies obtained in our simulation lead to the conclusion that in spherical particles, there is a surface layer with much higher degree of magnetic disorder than the core, which is the Ising version of the random canting of surface spins occurring in several fine particle with spinel structure. ${ }^{1-5}$ As opposite with the suggestion given by some authors ${ }^{14,17}$ that below a certain freezing temperature the surface layer enters a spin-glass-like state, our model does not give any indication of this phenomenology at any of the studied sizes and temperatures. Furthermore, the surface layer, by partially breaking the ferrimagnetic correlations, diminishes the zero-field $M_{\text {total }}$ but, at the same time, enhances $M_{\text {total }}$ at moderate fields. Although the surface is easily thermally demagnetized and easily magnetized by the field than the core, it does not behave as a dead layer, since, at any $T$, it is magnetically coupled to the core. All these facts put forward that the surface has higher magnetic response than the core, excluding a spin-glass freezing. Moreover, we do not observe irreversibilities between field and zero-field cooled magnetization curves, which is a key signature that in the scope of our model, neither finite-size or surface effects, nor the inclusion of surface vacancies are enough to account for the postulated spin-glass-like state.

Finally, let us mention that our model does not reproduce the experimentally observed shift of the hysteresis loops under field cooling adduced as a prove of the existence of the spin-glass-like state at the surface. ${ }^{14,24,17}$ Only when $h_{\mathrm{FC}}$ smaller than irreversibility fields $h_{\text {irr }}$ are used in the numerical experiment, hysteresis loops that are apparently shifted are obtained, which in fact are minor loops. In any case, the absence of this phenomenology is in agreement with the nonobservation of a spin-glass-like state at the surface, indicating that other ad hoc ingredients must be included in the model. For instance, enhanced surface anisotropy or exchange constants at the surface different than at the bulk, as is the case in exchange coupled multilayers. ${ }^{46,47}$ Current work is under progress to elucidate the possible influence of these new ingredients and of interparticle interactions.

\section{ACKNOWLEDGMENTS}

The authors are indebted to Professor Fèlix Ritort for close collaboration in the initial steps of this study and critical reading of the manuscript. We acknowledge CESCA and CEPBA under coordination of $C^{4}$ for the computer facilities. This work has been supported by SEEUID through Project No. MAT2000-0858 and CIRIT under Project No. 2000 SGR00025.
${ }^{1}$ J. M. D. Coey, Phys. Rev. Lett. 27, 1140 (1971).

${ }^{2}$ A. H. Morrish and K. Haneda, J. Appl. Phys. 52, 2496 (1981).

${ }^{3}$ R. H. Kodama, A. E. Berkowitz, E. J. McNiff, Jr., and S. Foner, Phys. Rev. Lett. 77, 394 (1996).

${ }^{4}$ D. Lin, A. C. Nunes, C. F. Majkrzak, and A. E. Berkowitz, J. Magn. Magn. Mater. 145, 343 (1995).

${ }^{5}$ J. Z. Jiang, G. F. Goya, and H. R. Rechenberg, J. Phys.: Condens. Matter 11, 4063 (1999).

${ }^{6}$ F. Gazeau, E. Dubois, M. Hennion, R. Perzynski, and Yu. L. Raikher, J. Magn. Magn. Mater. 40, 575 (1997).

${ }^{7}$ F. Gazeau, J. C. Bacri, F. Gendron, R. Perzynski, Yu. L. Raikher, V. I. Stepanov, and E. Dubois, J. Magn. Magn. Mater. 186, 175 (1998).

${ }^{8}$ A. Ochi, K. Watanabe, M. Kiyama, T. Shinjo, Y. Bando, and T. Takada, J. Phys. Soc. Jpn. 50, 2777 (1981).

${ }^{9}$ T. Okada, H. Sekizawa, F. Ambe, and T. Yamada, J. Magn. Magn. Mater. 31-34, 105 (1983).

${ }^{10}$ K. Haneda and A. H. Morrish, J. Appl. Phys. 63, 4258 (1988).
${ }^{11}$ Q. A. Pankhurst and R. J. Pollard, Phys. Rev. Lett. 67, 248 (1991).

${ }^{12}$ F. T. Parker, M. W. Foster, D. T. Margulies, and A. E. Berkowitz, Phys. Rev. B 47, 7885 (1993).

${ }^{13}$ S. Linderoth, P. V. Hendriksen, F. Bodker, S. Wells, K. Davis, S. W. Charles, and S. Mørup, J. Appl. Phys. 75, 6583 (1994).

${ }^{14}$ R. H. Kodama, S. A. Makhlouf, and A. E. Berkowitz, Phys. Rev. Lett. 79, 1393 (1997).

${ }^{15}$ M. P. Morales, C. J. Serna, F. B $\phi$ dker, and S. Mørup, J. Phys.: Condens. Matter 9, 5461 (1997).

${ }^{16}$ M. García del Muro, X. Batlle, and A. Labarta, Phys. Rev. B 59, 13584 (1999).

${ }^{17}$ B. Martínez, X. Obradors, Ll. Balcells, A. Rouanet, and C. Monty, Phys. Rev. Lett. 80, 181 (1998).

${ }^{18}$ T. Jonsson, J. Mattsson, C. Djurberg, F. A. Khan, P. Nordblad, and P. Svedlindh, Phys. Rev. Lett. 75, 4138 (1995).

${ }^{19}$ T. Jonsson, P. Nordblad, and P. Svedlindh, Phys. Rev. B 57, 497 (1998). 
${ }^{20}$ X. Batlle, M. García del Muro, and A. Labarta, Phys. Rev. B 55, 6440 (1997).

${ }^{21}$ J. L. Dormann, R. Cherkaoui, L. Spinu, M. Nogués, F. Lucari, F. D’Orazio, D. Fiorani, A. García, E. Tronc, and J. P. Jolivet, J. Magn. Magn. Mater. 187, L139 (1998).

${ }^{22}$ S. Mørup and E. Tronc, Phys. Rev. Lett. 72, 3278 (1994).

${ }^{23}$ P. Jonsson and P. Nordblad, Phys. Rev. B 62, 1466 (2000).

${ }^{24}$ R. H. Kodama and A. E. Berkowitz, Phys. Rev. B 59, 6321 (1999).

${ }^{25}$ H. Kachkachi, A. Ezzir, M. Nogués, and E. Tronc, Eur. Phys. J. B 14, 681 (2000).

${ }^{26}$ H. Kachkachi, M. Nogués, E. Tronc, and D. A. Garanin, J. Magn. Magn. Mater. 221, 158 (2000).

${ }^{27}$ H. Kachkachi and D. Garanin, J. Phys: Condens. Mater. (to be published).

${ }^{28}$ K. N. Trohidou and J. A. Blackman, Phys. Rev. B 41, 9345 (1990).

${ }^{29}$ X. Zianni and K. N. Trohidou, J. Appl. Phys. 85, 1050 (1999).

${ }^{30}$ D. A. Dimitrov and G. M. Wysin, Phys. Rev. B 50, 3077 (1994).

${ }^{31}$ D. A. Dimitrov and G. M. Wysin, Phys. Rev. B 51, 11947 (1995).

${ }^{32}$ K. Binder and A. P. Young, Rev. Mod. Phys. 58, 801 (1986).

${ }^{33}$ C. M. Soukoulis, K. Levin, and G. S. Grest, Phys. Rev. Lett. 48, 1756 (1982); C. M. Soukoulis, G. S. Grest, and K. Levin, ibid. 50, 80 (1983).

${ }^{34}$ D. P. Landau, Phys. Rev. B 14, 255 (1976).

${ }^{35}$ M. N. Barber, in Phase Transitions and Critical Phenomena, ed- ited by C. Domb and J. L. Lebowitz (Academic Press, New York, 1983), Vol. 8, p. 145.

${ }^{36}$ H. E. Stanley, Introduction to Phase Transitions and Critical Phenomena (Oxford University Press, New York, 1987).

${ }^{37}$ B. Martínez and R. E. Camley, J. Phys.: Condens. Matter 4, 5001 (1992); G. Xiao and C. L. Chien, J. Appl. Phys. 61, 4314 (1987); A. Corciovei, Phys. Rev. 130, 2223 (1963).

${ }^{38}$ J.-H. Park, E. Vescovo, H.-J. Kim, C. Kwon, R. Ramesh, and T. Venkatesan, Phys. Rev. Lett. 81, 1953 (1998).

${ }^{39}$ D. Zhao, F. Liu, D. L. Huber, and M. G. Lagally, Phys. Rev. B 62, 11316 (2000).

${ }^{40} \mathrm{H}$. Maletta, in Excitations in Disordered Solids, edited by M. Thorpe (Plenum, New York, 1981).

${ }^{41}$ E. C. Stoner and E. P. Wohlfarth, Philos. Trans. R. Soc. London, Ser. A 240, 599 (1948); IEEE Trans. Magn. 27, 3475 (1991).

${ }^{42}$ P. Gaunt, J. Appl. Phys. 59, 4129 (1986); Philos. Mag. B 48, 261 (1983).

${ }^{43}$ E. Viitala, J. Merikowski, M. Manninen, and J. Timonen, Phys. Rev. B 55, 11541 (1997).

${ }^{44}$ Z. X. Tang, C. M. Sorensen, K. J. Klabunde, and G. C. Hadjipanayis, Phys. Rev. Lett. 67, 3602 (1991).

${ }^{45}$ D. H. Han, J. P. Wang, and H. L. Luo, J. Magn. Magn. Mater. 136, 176 (1994).

${ }^{46}$ P. Milténi, M. Gierlings, J. Keller, B. Beschoten, G. Güntherodt, U. Nowak, and K. D. Usadel, Phys. Rev. Lett. 84, 4224 (2000).

${ }^{47}$ R. L. Stamps, J. Phys. D 33, R247 (2000). 